\section{An objective test of goodness of fit of the continuous strength model in STM*}

\author{
LARRY E, WOOD \\ University of Iowa, Iowa City, Iowa 52240
}

The continuous strength model of recognition memory was evaluated in a task where Ss were tested for recognition of 10 -number lists using a rating procedure. Maximum likelihood estimates of the parameters of the model were obtained by an iterative method on a high-speed computer, and a chi-square goodness-of-fit test was performed for individual Ss. For 15 of $20 \mathrm{Ss}$, the chi-square values were nonsignificant, $p<.05$, indicating that the model provided a good fit to the data. Although the model gave a good fit to the data, the $\Delta \mathrm{m}$ measure of sensitivity was highly correlated with a true recognition score computed by subtracting false alarms from correct recognitions.

A recurrent problem in the study of recognition memory has been the treatment of response bias both between $S$ s in a particular experimental condition and across conditions. Egan (1958) pointed out that traditional methods for correcting for response bias implicitly assume a threshold model of the underlying memory trace, and as an alternative he proposed a continuous strength model based on the theory of signal detection from sensory psychophysics (cf. Green \& Swets, 1966). Because the two types of models predict different functional relationships between hits and false alarms, it is possible to arrive at opposing conclusions about the effect of an independent variable, depending upon which model is used to account for response bias (cf. Freund, Brelsford, \& Atkinson, 1969). Since its introduction into the field of memory, the continuous strength model has become increasingly popular and has been used in the investigation of a variety of memory phenomena. The assumptions, applications, uses, and misuses of the continuous strength model as applied to memory are outlined in two recent reviews (Banks, 1970; Lockhart \& Murdock, 1970).

While the continuous strength model has been generally accepted as more appropriate than threshold models for evaluating recognition memory data, its usefulness has been restricted by the lack of adequate objective methods for estimating the necessary parameters and determining the goodness-of-fit of the model. Parameter estimates have typically been obtained from memory operating characteristic (MOC) curves which have

*The research was conducted while the author held an NDEA Title IV predoctoral fellowship from the Department of Health, Education and Welfare The author wishes to express his appreciation to Donald D. Dorfman, Michael D. Biderman, and James V. Hinrichs for their many helpful comments and suggestions. been fit by eye through plots of hits and false alarms on normal-normal coordinates. The fit of the model has been accepted if the data points did not deviate systematically from a straight line, although what constitutes a systematic deviation has usually not been specified. The major reason for this state of affairs, as pointed out by Green and Swets (1966, p. 404), is that until very recently optimal statistical procedures for fitting curves to data having variation on both the $x$ and $y$ coordinates had not been developed. An objective method of parameter estimation which yields estimates suitable for a chi-square test of goodness-of-fit is the method of maximum likelihood, although its use with the continuous strength model entails the solution of a system of nonlinear simultaneous equations. Recently, however, Dorfman and Alf (1968, 1969) have developed procedures for obtaining maximum likelihood estimates of the parameters of signal detection theory for both yes-no data and rating method data, using methods of numerical analysis on a high-speed computer. They have suggested that the chi square based on maximum likelihood estimates (MLEs) should provide a sensitive index of goodness-of-fit with which to compare various models of the data of receiver operating characteristic curves.

The major purpose of the present study was to evaluate the fit of the continuous strength model to some recognition memory data with a chi-square test of goodness-of-fit using Dorfman and Alf's (1969) procedure to obtain maximum likelihood estimates of the parameters. The paradigm used in the experiment tested recognition memory for 10 -number lists and is one to which the continuous strength model has not been applied. A rating method procedure was used because of the efficiency it affords in generating data necessary for estimating the parameters of the model.
Subjects

The Ss were 22 students from the introductory psychology courses at the Universty of Iowa; they were tested in groups of four.

\section{Materials}

The stimulus materials were lists composed of 10 of the numbers 1-20 inclusive. In all, 60 lists were constructed, using a table of random permutations of the 20 numbers so that each number appeares: no more than once, if at all, in each list.

\section{Procedure}

The stimulus lists were presented via a tape recorder at the rate of $1 \mathrm{item} / \mathrm{sec}$. The Ss were given answer sheets containing rows of the numbers, 1-20, with a box beneath each number. Following the presentation of each list, the Ss were required to designate which 10 of the 20 numbers had been presented by placing a plus sign in the boxes under the numbers that had been presented and a minus sign for those that had not been presented. In addition to making a binary decision, Ss were further required to rate their confidence in their decisions. A rating of " 3 " meant very confident, " 1 " meant very little confidence, and " 2 " was for an intermediate level of confidence. In writing their answers, Ss were instructed to always begin with the number " 1 " and proceed across the page, filling in all the boxes as they went. Approximately $1 \mathrm{~min}$ was allowed for writing the answers for each list. The 60 lists were presented in three blocks of 20 lists each, with a 5 -min rest period between blocks. With 20 responses per list, each $S$ contributed a total of 1,200 responses.

\section{RESULTS AND DISCUSSION}

The data from two Ss were discarded because of their failure to use all of the rating categories. Probabilities for estimating parameters were obtained for the remaining $20 \mathrm{Ss}$ by assuming that with $k$ rating categories, $S$ sets $k-1$ cutoffs on the decision axis, and whenever an item exceeds the strength value of the $j$ th cutoff, it is given a rating of $j+1$. In the present study, a rating of " -3 " represents the low and of the continuum, whereas " +3 " is at the high end. The relevant probabilities are then $P\left(R_{j} \mid S_{2}\right)$ and $P\left(R_{j} \mid S_{1}\right)$, where $R_{j}$ is a rating of $j, S_{2}$ refers to the 10 numbers that were presented on a particular trial, and $S_{1}$ refers to those that were not. It was assumed that an item accepted at a particular criterion would have been accepted at all lower criteria. For each 
Table 1

Parameter Estimates and $x^{2}$ Values from Goodness-of-Fit Tests

\begin{tabular}{|c|c|c|c|c|c|c|c|}
\hline \multirow{2}{*}{$\begin{array}{c}\text { S } \\
\text { No. }\end{array}$} & \multicolumn{3}{|c|}{ Least-Squares Estimates } & \multicolumn{3}{|c|}{ Maximum Likelihood Estimates } & \multirow[b]{2}{*}{$\Delta \mathbf{m}$} \\
\hline & a & $\mathbf{b}$ & $x^{2}(d f=3)$ & $\mathbf{a}$ & $\mathbf{b}$ & $x^{2}(d f=3)$ & \\
\hline 4 & 1.050 & .715 & .807 & 1.043 & .712 & .417 & 1.465 \\
\hline 20 & 1.097 & .718 & 1.076 & 1.095 & .718 & .594 & 1.524 \\
\hline 1 & .958 & .726 & 2.360 & .951 & .727 & .890 & 1.308 \\
\hline 7 & 1.287 & .775 & 3.570 & 1.297 & .788 & 1.557 & 1.647 \\
\hline 21 & .885 & .693 & 4.771 & .880 & .685 & 1.942 & 1.285 \\
\hline 13 & .962 & .590 & 2.959 & .972 & .587 & 1.979 & 1.656 \\
\hline 5 & 1.091 & .821 & 6.010 & 1.080 & .812 & 2.655 & 1.330 \\
\hline 18 & .822 & .973 & 6.028 & .861 & .871 & 2.720 & 1.061 \\
\hline 15 & 1.101 & .617 & 5.687 & 1.103 & .618 & 3.599 & 1.787 \\
\hline 11 & 1.067 & .698 & $14.650 * *$ & 1.077 & .703 & 3.698 & 1.530 \\
\hline 2 & 1.047 & .812 & 6.314 & 1.044 & .811 & 3.897 & 1.287 \\
\hline $\mathbf{9}$ & 1.249 & .627 & $9.666^{*}$ & 1.236 & .626 & 4.245 & 1.974 \\
\hline 8 & 1.221 & .697 & $12.512 * *$ & 1.231 & .703 & 4.683 & 1.752 \\
\hline 12 & 1.249 & .732 & $14.263^{* *}$ & 1.248 & .732 & 5.713 & 1.706 \\
\hline 19 & 1.168 & .655 & $14.500^{* *}$ & 1.520 & .643 & 7.801 & 1.817 \\
\hline 14 & 1.486 & .702 & $14.888 * *$ & 1.476 & .619 & $10.133^{*}$ & 2.384 \\
\hline 3 & 1.281 & .698 & $28.628 * * *$ & 1.260 & .690 & $15.187 * *$ & 1.827 \\
\hline 17 & .969 & .729 & $23.909 * * *$ & .955 & .725 & $16.645 * * *$ & 1.024 \\
\hline 16 & 1.131 & .892 & $44.643 * * *$ & 1.132 & .896 & $18.535 * * *$ & 1.263 \\
\hline 6 & .819 & .774 & $69.854^{* * *}$ & .884 & .794 & $22.021 * * *$ & 1.113 \\
\hline
\end{tabular}

the chi-square values for the LSEs were approximately twice as large in each case as those based on MLEs, so that 10 of the 20 were significant, $p<.05$. The sums of the individual chi squares were 277.095 and 128.911, respectively, for the LSEs and MLEs.

As pointed out previously, the $d^{\prime}$ measure assumes that the slope parameter, $\mathrm{b}$, has the value of 1 . In view of the present data, this assumption is clearly not tenable. As Table 1 indicates, the values range from .587 to .896 , indicating that $\sigma_{1}<\sigma_{2}$ in all cases. Because of the differences in $b$ across Ss, it would seem to be more appropriate to use the measures which take unequal variance into account, such as $\Delta \mathrm{m}$ or $\mathrm{d}_{\mathrm{c}}^{\prime}$ (cf. Green \& Swets, 1966).

Memory operating characteristic curves plotted on normal-normal coordinates are presented in Fig. 1 for the six Ss with largest chi squares and in Fig. 2 for the six
$* p<.05, * * p<.01, * * * p<.001$

cutoff, the rating probabilities were then cumulated from $j$ to $k$, making each the probability of $R_{j}$ or greater. When the cumulative probabilities are transformed into standard normal deviates and plotted on normal-normal coordinates, the MOC curve should be a linear function of the form $Y_{k}=a-b Z_{k}$, where $\mathrm{a}=\left(\begin{array}{lll}\mu_{2} & -\mu_{1}\end{array}\right) / \sigma_{2}, \quad \mathrm{~b}=\sigma_{1} / \sigma_{2}$, $\mathrm{Z}_{\mathrm{k}}=\mathrm{x}_{\mathrm{k}}-\mu_{1} / \sigma_{1}$, and $\mathrm{x}_{\mathrm{k}}$ is a particular cutoff. Following the conventions proposed by Green and Swets (1966), the MOC curve may be summarized by $\mathrm{D}(\Delta \mathrm{m}, \mathrm{s})$, where $\Delta \mathrm{m}=\mathrm{a} / \mathrm{b}$ and $\mathrm{s}=\mathrm{b}$. When $\sigma_{2} / \sigma_{1}=1, \Delta \mathrm{m}=\mathrm{d}^{\prime}=\mathrm{a}$.

Maxinum likelihood estimates for the parameters of the model were obtained for each $S$ using the procedure developed by Dorfman and Alf (1969) for rating method data. Since the procedure involves a method of successive approximation, initial estimates of the parameters were needed. These were obtained with the method of least squares, and, for comparison purposes, chi squares were computed for both the MLEs and the least-squares estimates (LSEs). Table 1 lists the estimates of $a$ and $b$ and the chi-square values for both the LSEs and MLEs, as well as the values for $\Delta \mathrm{m}$ based on the MLEs. As indicated in the table, the chi-square values based on MLEs were nonsignificant, $p>.05$, for 15 of the 20 Ss. Given the large number of observations, it seems reasonable to conclude that the model provides a good fit to the data. The sensitivity of the chi-square test is indicated by a comparison of the values obtained witl the two estimation procedurcs. The estimates of $a$ and $b$ for the same $\mathrm{S}$ differed only in the third or fourth significant digit in most cases; yet

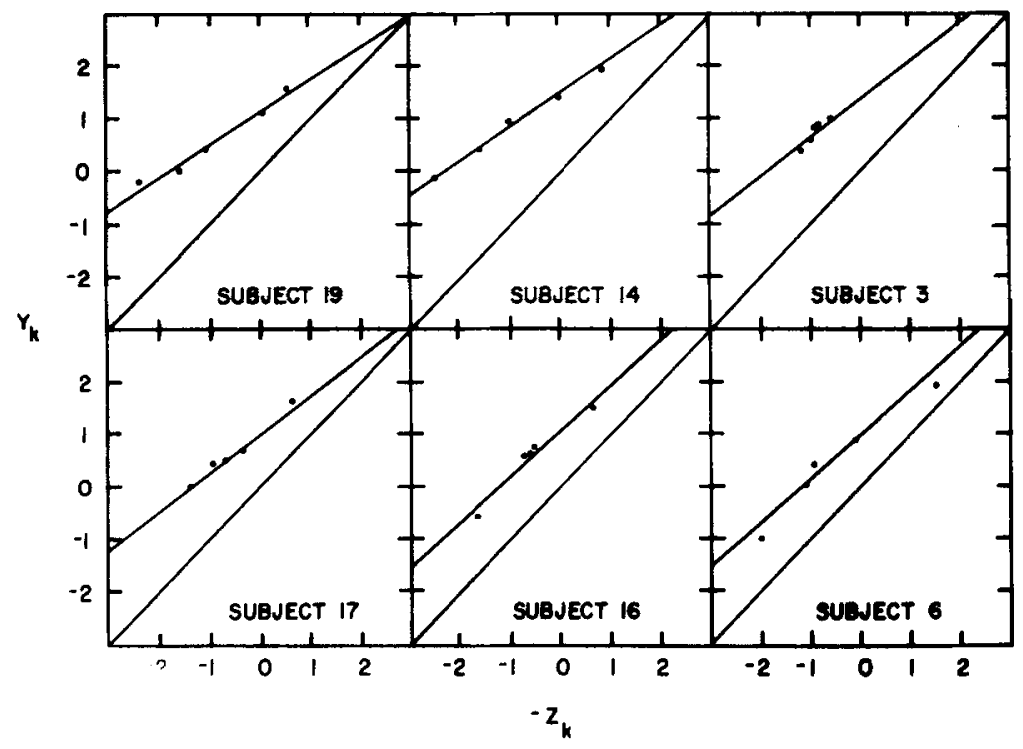

Fig. 1. MOC curves for the Ss with largest chi squares.

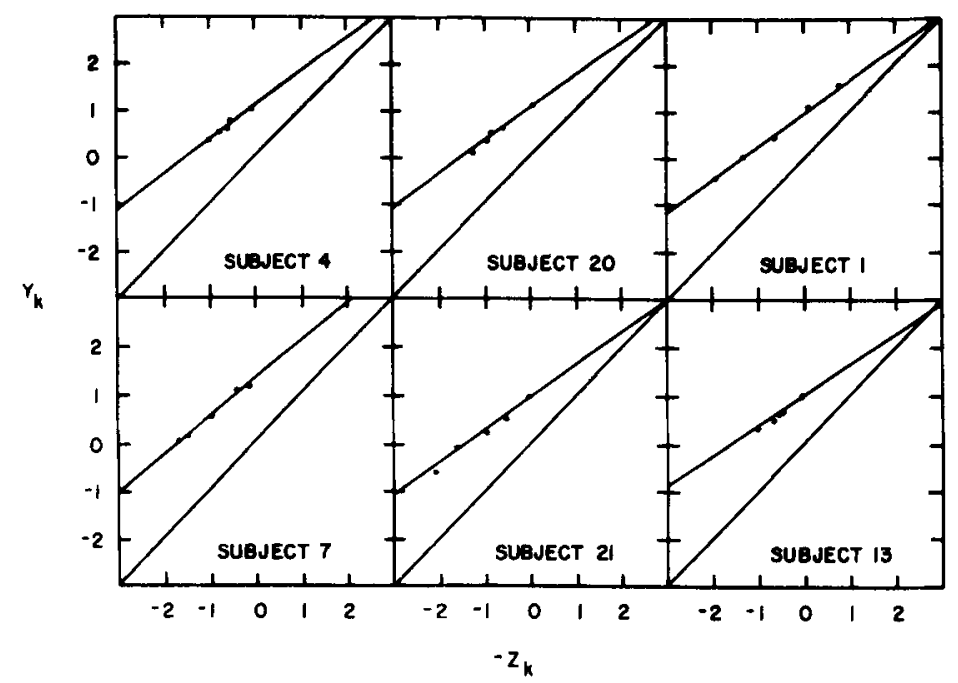

Fig. 2. MOC curves for the $S$ s with smallest chi squares. 


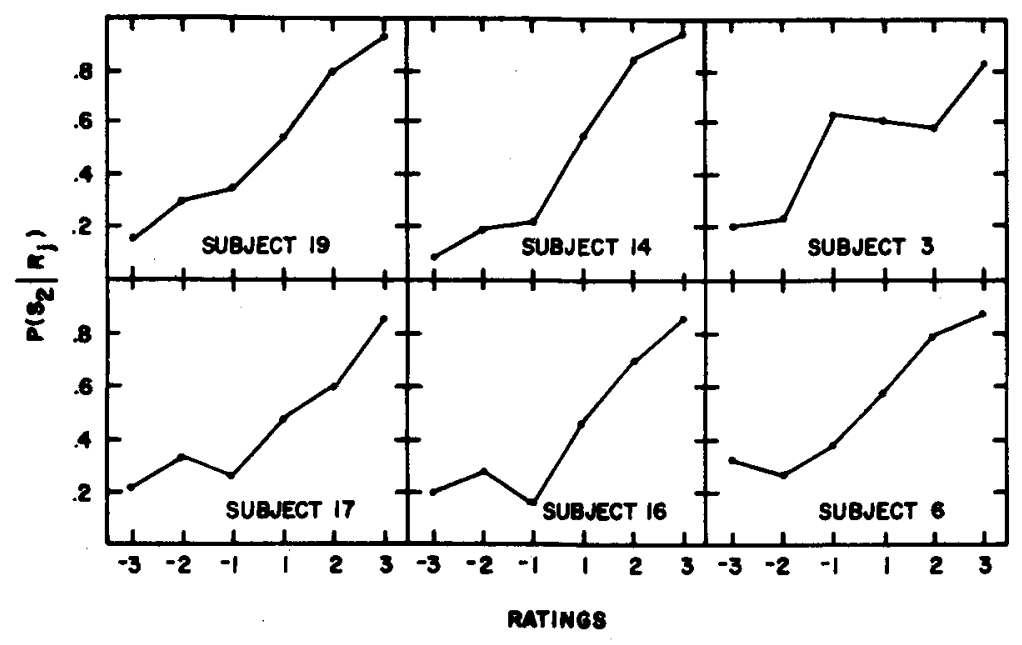

Fig. 3. A posteriori probabilities for the Ss with largest chi squares.

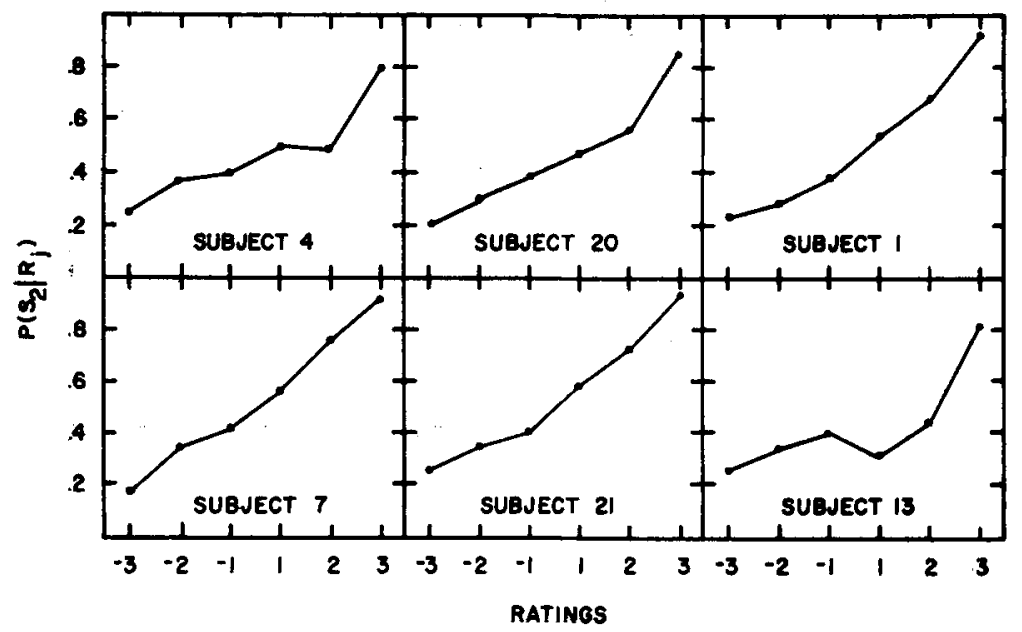

Fig. 4. A posteriori probabilities for the Ss with smallest chi squares.

Ss with smallest chi squares. The most striking feature of the two sets of curves is the lack of obvious differences between them. This serves to point out the inadequacy of fitting curves by eye, both for obtaining parameter estimates and for determining the goodness-of-fit of the model.

One strong implication of a continuous strength model is that a posteriori probabilities for items presented, $P\left(S_{2} \mid R_{j}\right)$, should show an orderly increase across ratings from " -3 " to " +3 ." The a posteriori probabilities for those six Ss with the largest chi squares and those six with smallest chi squares are plotted in Figs. 3 and 4, respectively. In general, the recognition memory, it seems important to employ a model which best describes the functional relationship between hits and false alarms regardless of the underlying processes by which it is generated. On the other hand, it could be argued that results obtained from estimates of $\Delta \mathrm{m}$ and traditional methods of accounting for response bias are not discrepant enough to warrant the extra investment necessary to obtain parameter estimates for the continuous strength model. In order to obtain a rough indication of the discrepancy between the two measures from the present data, a true recognition score was obtained for each $S$ by subtracting the proportion of false alarms from the proportion of correct recognitions with the data collapsed across ratings. A product-moment correlation was then computed between the true recognition score and the value of $\Delta m$ for each $S$. The resulting correlation coefficient was $r=895$, which was significantly different from zero, $t=26.93$, $\mathrm{df}=18, \mathrm{p}<.001$. The correlation is rather high, indicating that in the present case the two measures seem to convey similar information.

\section{REFERENCES}

BANKS, W. P. Signal detection theory and human memory. Psychological Bulletin, 1970, 74, 81-99.

DORFMAN, D. D., \& ALF, E., JR. Maximum likelihood estimation of parameters of signal detection theory-A direct solution. Psychometrika, 1968, 33, 117-124.

DORFMAN, D. D., \& ALF, E., JR. Maximum-likelihood estimation of parameters of signal detection theory and determination of confidence intervals-Rating method data. Journal of Mathematical Psychology, 1969, 6, 487-496.

EGAN, J. P. Recognition memory and the operating characteristic. Technical Note AFCR-TN-58-51, Hearing and Communication Laboratory, Indiana University, 1958.

FREUND, R, D.. BRELSFORD, J. W., \& ATKINSON, R. C. Recognition vs recall: Storage or retrieval differences? Quarterly Journal of Experimental Psychology, 1969. 21, 214-224.

GREEN, D. M., \& SWETS, J. A. Signal detection theory and psychophysics. New York: Wiley, 1966.

LOCKHART. R. S., \& MURDOCK, B. B., JR. Memory and the theory of signal detection. Psychological Bulletin. 1970, 74, 100-109.

(Accepted for publication October 18, 1970.) 